\title{
Aspectos Psicossociais na Era dos Novos Tratamentos da AIDS
}

\author{
Eduardo Augusto Remor ${ }^{1}$ \\ Universidad Autónoma de Madrid
}

\begin{abstract}
RESUMO: O presente artigo descreve o impacto no bem-estar (ansiedade e depressão) e qualidade de vida das pessoas HIV+ quando o tratamento anti-retroviral não apresenta os resultados esperados. Foram avaliadas, através de questionários, as percepções e expectativas com o tratamento anti-retroviral, o nível de ansiedade e depressão, o nível de apoio social e a qualidade de vida percebida em 80 pacientes HIV+ em atendimento em um hospital universitário. Os resultados indicaram que as percepções e expectativas negativas com o tratamento podem modular o estado emocional e impactar negativamente a qualidade de vida das pessoas HIV+; e que um nível de apoio social adequado é capaz de atenuar este impacto negativo.
\end{abstract}

Palavras-chave: HIV; AIDS; aspectos psicológicos; hemofilia; tratamento anti-retroviral.

\section{Psychosocial Issues in the Era of New AIDS Treatment}

\begin{abstract}
The present article describes the impact in the well-being (anxiety and depression) and quality of life of HIVpositive people when antiretroviral treatment doesn't present the expected results. Perceptions and expectations regarding antiretroviral treatment, anxiety and depression levels, social support levels and perceived quality of life were assessed by questionnaires. Eighty HIV-positive patients were assessed at the university hospital. Results indicate that both negative perceptions and expectations about treatment can modulate the emotional state and have a negative impact on the quality of life of the HIV-positive people. Adequate levels of social support have also a buffering effect on negative outcomes.
\end{abstract}

Key words: HIV; AIDS; psychological aspects; hemophilia; antiretroviral treatment.

Nos últimos anos tem-se observado um aumento na compreensão da patogenia da infeção por HIV, resultando em uma mudança drástica no tratamento desta. Novos e mais poderosos medicamentos foram introduzidos e sua eficácia foi demonstrada através de ensaios clínicos, indicando que os tratamentos combinados atrasam a progressão do HIV e melhora a sobrevivência. Nos países industrializados observou-se um declínio na incidência das doenças oportunistas e a mortalidade por Aids diminuiu (Guardiola \& Soriano, 2000).

No mesmo sentido, desde o principio da epidemia da Aids, houve um grande esforço por parte dos pesquisadores e clínicos em entender o impacto psicossocial da infeção por HIV naqueles que vivem com o vírus (Catalan, Meadows \& Douzenis, 2000; Ferrando, 1998; Kelly, Otto-Salaj, Sikkema, Pinkenton \& Bloom, 1998; Ulla \& Remor, 2002). Em 1996 a introdução dos novos tratamentos antiretrovirais, denominados HAART (Highly Active Antiretroviral Theraphy), possibilitou uma mudança na perspectiva e prognóstico da Aids, gerando uma onda de otimismo nas pessoas que vivem com o HIV.

Porém, recentemente, alguns estudos demostraram que apesar da manutenção de uma carga viral indetectável por 20 meses ou mais, o vírus persiste "adormecido" nos gânglios linfáticos e células mononucleares no sangue periférico, podendo reativar-se no caso de suspensão do tratamento (Furtado, Callaway \& Phair, 1999). Ademais, uma porcentagem

1 Endereço: Dept. Psicología Biológica y de la Salud (Desp. 28), Facultad de Psicología, Universidad Autónoma de Madrid, Ciudad Universitaria de Cantoblanco, 28049, Madrid. España. importante dos pacientes sob tratamento HAART experimentou efeitos secundários importantes como resultado do tratamento, produzindo um impacto negativo em sua qualidade de vida (Siegel, Schrimshaw \& Dean, 1999). Outros, ainda, se encontraram frente a um fracasso terapêutico gerado pela resistência do vírus ao tratamento (Nájera, 1997). Estes efeitos colaterais trouxeram novamente a incerteza para muitos dos infectados.

O presente trabalho pretende descrever, desde a perspectiva do paciente, o impacto e as preocupações destes, e sua relação com o mal-estar psicológico, como resultado das dificuldades experimentadas com a terapia HAART.

\section{Método}

\section{Sujeitos}

Oitenta pacientes $\mathrm{HIV}+$, dos quais 40 haviam sido infectados por transfusão de hemoderivados (Hemofílicos e Von Willebrand, Grupo 1) e 40 por via sexual ou compartilhamento de seringas (Grupo 2), constituíram a amostra. Os primeiros foram derivados pelo Serviço de Hematologia e Hemoterapia do Hospital Universitário La Paz (Madri), enquanto que os segundos foram derivados pelo Serviço de Medicina Interna, Unidade de HIV/AIDS, deste mesmo hospital.

Ambos os grupos foram compostos por pacientes com mais de 10 anos de sobrevivência desde o momento da infecção (long-term survival), todos eles sob tratamento combinado de terapia anti-retroviral e inibidores da transcriptase e protease - conhecida como HAART. 
A média de idade dos pacientes do Grupo 1 foi de 36 anos (variando de 26 a 50 anos) e a dos pacientes do Grupo 2 de 29 anos (variando de 18 a 47 anos). Com respeito ao sexo, $95 \%$ dos pacientes do Grupo 1 foram homens e $5 \%$ mulheres, enquanto que, no Grupo 2, 67,5\% dos pacientes foram homens e 32,5\% mulheres. Em relação às características clínicas e imunológicas, a tabela 1 descreve a composição da amostra.

Tabela 1. Características clínicas e imunológicas da amostra.

\begin{tabular}{lcc}
\hline & Grupo 1 $(\boldsymbol{n}=\mathbf{4 0})$ & Grupo 2 $(\boldsymbol{n}=\mathbf{4 0})$ \\
\hline Classificação CDC: & & \\
A1, A2, A3 & $42 \%$ & $20 \%$ \\
B1, B2, B3 & $34 \%$ & $32,5 \%$ \\
C1, C2, C3 & $24 \%$ & $47,5 \%$ \\
\hline Linfócitos T CD4: & & \\
Média & 273 & 296 \\
Desvio Padrão & 187,64 & $10-1016$ \\
Intervalo & $8-899$ & 222190 \\
\hline Carga Viral: & & 607495,33 \\
Média & 35414,82 & $0-2670000$ \\
Desvio Padrão & 60014,66 & \\
Intervalo & $0-263000$ &
\end{tabular}

\section{Materiais e instrumentos}

Dados sócio-demográficos e clínicos foram obtidos a partir do prontuário médico dos pacientes. Os níveis de ansiedade e depressão foram avaliados por meio da escala HADS - Hospital Anxiety and Depression Scale, de acordo com Zigmond e Snaith (1983). Nesta escala, 14 itens subdivididos em duas subescalas de 7 itens cada avaliam os níveis de ansiedade e depressão, respectivamente. Trata-se de um instrumento auto-aplicado com uma escala de resposta de 0 a 3. Obtém-se uma pontuação independente para cada subescala, sendo que uma maior pontuação indica maiores níveis de ansiedade ou depressão.

O nível de apoio social foi avaliado por meio da aplicação do questionário de Apoio Social Funcional Duke-UNC11 (Broadhead, Gehlbach, Degruy \& Kaplan, 1988). Consta de 11 itens com um intervalo de resposta tipo likert, de 1 a 5 , onde uma maior pontuação indica menor apoio social.

A qualidade de vida percebida foi avaliada por meio da análise das respostas a seguinte pergunta: “Atualmente, como qualificaria a sua qualidade de vida?". Um intervalo de resposta tipo likert, de 1 a 5 , foi utilizado, onde 1 indicava "muito ruim" e 5 "muito boa". Esta pergunta demonstrou alta correlação com a qualidade de vida medida pelo questionário Medical Outcomes Study-SF30 em um estudo anterior (Remor, 2001), assegurando sua validade como indicador geral percebido da qualidade de vida. Finalmente, as percepções e expectativas sobre o tratamento foram avaliadas por meio de um questionário desenvolvido para este estudo. Este compreendeu 9 perguntas fazendo referencia ao grau de informação sobre o tratamento anti-HIV, expectativas positivas e otimismo frente aos resultados do tratamento, percepção de mudanças positivas ou negativas devido ao tratamento, bem como percepção de dificuldades e problemas decorrentes do tratamento anti-retroviral. As perguntas foram agrupadas em 4 subescalas decorrentes da análise fatorial de todos os items. A tabela 2 descreve o questionário e os dados psicométricos da escala.

\section{Procedimento}

Os participantes foram recrutados de forma consecutiva no Serviço de Hematologia e Hemoterapia, e no Serviço de Medicina Interna do Hospital. Suas participações foram voluntárias, e todos assinaram um consentimento informado para sua inclusão no estudo. Os critérios de inclusão foram ser maior de 18 anos de idade e HIV+. Os critérios de exclu-

Tabela 2. Questionário de percepções e expectativas sobre o tratamento antiretroviral (Traduzido ao português): Indicadores psicométricos (Análise fatorial, componentes principais: Varimax com Kaiser), $\mathrm{n}=80$

\begin{tabular}{|c|c|c|c|c|}
\hline \multicolumn{5}{|l|}{ Prova de Esfericidade de Barlet $[\times 2(36)=119,310 ; p=0,000]$} \\
\hline Fatores & 1 & 2 & 3 & 4 \\
\hline \multicolumn{5}{|l|}{ Expectativas e otimismo } \\
\hline - Quais são suas expectativas em relação a estes novos tratamentos? (1 muito ruins - 5 muito boas) & 0,79 & & & \\
\hline - Considera que os novos tratamentos podem repercutir positivamente na evolução da sua doença? (1 nada - 5 muito) & 0,86 & & & \\
\hline - Se encontra mais animado ou motivado com a aparição dos novos medicamentos? (1 nada - 5 muito) & 0,82 & & & \\
\hline $\begin{array}{l}\text { - Como estimaria a intensidade dos efeitos colaterais relacionados com a tomada dos anti-retrovirais? (1 muito } \\
\text { intensos - } 5 \text { nada intensos) }\end{array}$ & 0,64 & & & \\
\hline \multicolumn{5}{|l|}{ Percepção de dificuldades e problemas } \\
\hline - Alguma destas mudanças provocou-lhe algum tipo de problema? (1 nenhum - 5 bastante) & & 0,66 & & \\
\hline - Em que medida teve que refazer sua vida com o surgimento dos novos fármacos? (1 nada - 5 muito) & & 0,78 & & \\
\hline \multicolumn{5}{|l|}{ Percepção de mudanças } \\
\hline - O surgimento dos novos tratamentos acarretou algum tipo de mudança em sua vida? (1 não nenhum - 5 sim, muitos) & & & 0,76 & \\
\hline - Considera que sua saúde melhorou desde que começou a tomar os anti-retrovirais? (1 nada - 5 muito) & & & 0,74 & \\
\hline \multicolumn{5}{|l|}{ Informação } \\
\hline - Como avalia a informação que possui sobre os anti-retrovirais? (1 nenhuma - 5 muita) & & & & 0,93 \\
\hline
\end{tabular}

Nota: Variância total explicada dos 4 fatores: $73,1 \%$; Prova de esfericidade de Barlet $\left[\mathrm{x}^{2}(36)=119,310 ; p=0,000\right]$. 
Tabela 3. Descrição das percepções e expectativas sobre o tratamento antiretroviral considerando a mostra total e os diferentes grupos.

\begin{tabular}{|c|c|c|c|c|}
\hline Categorias & T $(n=80)$ & G1 $(n=40)$ & G2 $(n=40)$ & Contraste (G1 x G2) \\
\hline Expectativas e optimismo & $14,7 \pm 2,3[10-20]$ & $15,4 \pm 2,1$ & $14,0 \pm 2,3$ & $t=2,568 ; p=0,01$ \\
\hline Percepção de dificuldades e problemas & $3,8 \pm 1,6[2-9]$ & $3,7 \pm 1,4$ & $3,9 \pm 1,7$ & $t=-0,425 ; p=0,67$ \\
\hline Percepção de mudanças & $5,9 \pm 1,6[2-10]$ & $5,5 \pm 2,2$ & $6,3 \pm 1,2$ & $t=-2,011 ; p=0,05$ \\
\hline Informação & $3,2 \pm 1,0[1-5]$ & $3,5 \pm 1,0$ & $3,0 \pm 1,0$ & $t=2,082 ; p=0,04$ \\
\hline
\end{tabular}

Tabela 4. Comparação entre os Grupos 1 e 2 considerandos as variáveis psicossociais.

\begin{tabular}{|c|c|c|c|}
\hline \multicolumn{4}{|c|}{$t$ de Student para mostras independentes } \\
\hline Categorias & G1 $(n=40)$ & G2 $(n=40)$ & Contraste (G1 x G2) \\
\hline Ansiedade & $5,5 \pm 4,9$ & $8,8 \pm 3,9$ & $t=-3,265 ; p=0,002$ \\
\hline Apoio social (maior pontuação indica menor apoio social) & $19,0 \pm 9,3$ & $26,0 \pm 10,3$ & $t=-3,150 ; p=0,002$ \\
\hline Qualidade de vida percebida & $3,9 \pm 0,7$ & $3,5 \pm 0,6$ & $t=2,226 ; p=0,02$ \\
\hline
\end{tabular}

Tabela 5. Relação entre percepções e expectativa sobre o tratamento e variáveis psicossociais.

\begin{tabular}{|c|c|c|c|}
\hline Categorias & Ansiedade & Depressão & QVp \\
\hline Expectativas e optimismo & $-0,383, p=0,001$ & $-0,335, p=0,005$ & $0,361, p=0,002$ \\
\hline Percepção de dificuldades e problemas & $0,294, p=0,02$ & N.S. & N.S. \\
\hline Percepção de mudanças & N.S. & N.S. & N.S. \\
\hline Informação & N.S. & N.S. & N.S \\
\hline
\end{tabular}

QVp - Qualidade de Vida percebida

são foram consumo de drogas nos últimos 6 meses e não aceitação do consentimento informado.

\section{Análise de dados}

Inicialmente, foram realizadas análises descritivas a fim de descrever as características sociodemográficas. A seguir, exploraram-se as diferenças entre os dois grupos de pacientes com diferentes vias de infecção por HIV (Hemofílicos e von Willebrand versus Infecção por via sexual e compartilhamento de seringas), dado que a bibliografia especializada indica existir uma diferença na evolução da infecção por HIV no grupo infectado por transfusão de hemoderivados (Hemofílicos e von Willebrand) devido a uma cepa viral menos virulenta (Vallely, Mani, Stoddart, Cleator \& Lucas, 1996). Para todas as comparações entre grupos empregouse o teste T de Student. O teste de correlação de Pearson foi utilizado para investigar a relação entre as variáveis psicossociais. Valores de $p<0,05$ foram considerados significativos.

\section{Resultados}

A comparação do perfil clínico e inmunológico dos pacientes HIV+, pertencentes ao grupo 1 (hemofilia e von Willebrand) e ao grupo 2 (infeção via sexual ou por intercâmbio de seringas), revelou que ambos grupos não se diferenciaram significativamente quanto ao número de linfócitos T CD4 $(p=0,62)$ e à carga viral $(p=0,06)$, apesar da diferença aparente entre os valores destes (tabela 1$)$. No que se refere ao estagio da doença (classificação CDC), este foi sig- nificativamente mais avançado no grupo $2(\mathrm{t}=-2,630 ; p=$ $0,01)$.

No que se refere às percepções e expectativas dos pacientes em relação ao tratamento anti-retroviral, observou-se diferenças entre os grupos. O Grupo 1 apresentou maior frequiência de expectativas positivas e otimismo frente ao tratamento, e maior informação auto-declarada sobre o tratamento médico em comparação ao Grupo 2. No entanto, foram os pacientes do Grupo 2 que perceberam maior freqüência de mudanças devido ao tratamento. Em relação às dificuldades e problemas percebidos não foram identificadas diferenças entre os grupos, conforme indicado na tabela 3.

No que refere às variáveis psicológicas observou-se diferenças significativas entre os grupos. O grupo 1 apresentou menor nível de ansiedade e depressão, maior nível de apoio social e melhor qualidade de vida percebida em relação ao Grupo 2. A tabela 4 descreve esses resultados.

Para verificar a relação entre as percepções e expectativas em relação ao tratamento anti-retroviral e as variáveis psicossociais (ansiedade, depressão e qualidade de vida percebida), realizou-se uma análise de correlação entre estas variáveis. A tabela 5 ilustra os valores da correlação de Pearson considerando a amostra total. Foram incluídos somente os valores significativos.

Com o objetivo de descrever o impacto da percepção de resultados negativos em relação ao tratamento HAART no estado emocional e na qualidade de vida percebida dos pacientes, realizou-se uma comparação alocando os pacientes em dois grupos: um grupo com aqueles pacientes que referiram ter maior informação sobre o tratamento anti-HIV, expectativas positivas e otimismo frente aos resultados do tra- 
tamento, maior percepção de mudanças devidas ao tratamento e ausência de dificuldades e problemas decorrentes do mesmo; e outro oposto, com menor informação, expectativas negativas e pessimismo, menor percepção de mudanças e vivência de dificuldades e problemas com o tratamento. Utilizou-se como ponto de corte para a determinação dos grupos a mediana de cada variável. Os resultados são apresentados na tabela 6 .

Por ultimo, considerando que o apoio social pode ser uma variável mediadora das percepções e expectativas sobre o tratamento, bem como do impacto emocional e da qualidade de vida percebida, realizou-se uma comparação entre os pacientes com maior e menor nível de apoio social, considerando as variáveis relacionadas com o tratamento, e as psicossociais. Determinou-se como ponto de corte, para a divisão dos grupos, a mediana do nível de apoio social.

Os resultados indicaram que, considerando o nível de apoio social, observou-se diferenças no que se refere à percepção de mudanças devido ao tratamento anti-HIV $(\mathrm{t}=$ $2,470 ; p=0,01)$, sendo o grupo com um maior apoio social percebido o que menos referiu mudanças em sua vida com o tratamento. Quanto ao estado emocional e à qualidade de vida, observou-se que os pacientes com um maior nível de apoio social experimentaram menor ansiedade $(\mathrm{t}=3,427 ; p$ $=0,001)$ e depressão $(\mathrm{t}=3,765 ; p=0,000)$ e maior qualidade de vida $(\mathrm{t}=-2,143 ; p=0,03)$.

\section{Discussão}

Uma série de resultados interessantes emergem a partir deste estudo. Em primeiro lugar, observou-se uma diferença significativa nas percepções e expectativas com o tratamento, estado emocional e qualidade de vida percebida considerando os dois grupos de pacientes: aqueles infectados por hemoderivados (hemofílicos e von Willebrand, Grupo 1) e aqueles infectados por via sexual ou compartilhamento de seringas (Grupo 2). Nos primeiros, observou-se uma maior adaptação e assimilação ao tratamento anti-retroviral, descrita pelo maior conhecimento sobre o tratamento, maior frequiência de expectativas positivas e otimismo frente ao tratamento e percepção de menores mudanças no cotidiano, se comparados aos do Grupo 2. Considerando que o Grupo 2 apresentou uma deterioração significativamente maior do que a do Grupo 1, é possível supor que o estado de saúde estaria modulando as diferentes percepções sobre o resultado do tratamento. Em outras palavras, aqueles que não experimentaram uma melhora significativa no processo da doença, conforme esperado pelo tratamento, tenderam a decepcionar-se e desmotivar-se com este, gerando expectativas negativas e maximizando as dificuldades e problemas (efeitos colaterais) decorrentes do tratamento.

Esta avaliação negativa, associada a uma saúde deteriorada e com possibilidades limitadas de tratamento, aumentaria o nível de ansiedade e depressão, refletindo de forma negativa na qualidade de vida dos pacientes. Antecipar e intervir sobre estes aspectos pode ser uma estratégia útil para prevenir a desesperança e o sofrimento emocional associados às complicações no tratamento da Aids. Ademais, estudos anteriores (Ortiz \& cols., 2000; Remor, 2000) demostraram que estas percepções podem dificultar a adesão ao tratamento, gerando um ciclo vicioso no qual as percepções negativas provocam uma diminuição ou interrup-

Tabela 6. Percepções e expectativas sobre o tratamento anti-retroviral: impacto sobre o estado emocional e qualidade de vida percebida

\begin{tabular}{|c|c|c|c|}
\hline \multicolumn{4}{|c|}{ Contraste ( $T$ de Student) } \\
\hline \multicolumn{4}{|c|}{ Expectativas e otimismo } \\
\hline \multicolumn{2}{|c|}{ Expectativas positivas e otimismo } & \multicolumn{2}{|c|}{ Expectativas negativas e pessimismo } \\
\hline Ansiedade & $5,4 \pm 3,4$ & $9,7 \pm 5,1$ & $-3,972 ; p=0,000$ \\
\hline Depressão & $2,5 \pm 3,0$ & $5,2 \pm 4,1$ & $-3,160 ; p=0,002$ \\
\hline Qvp & $4,0 \pm 0,5$ & $3,4 \pm 0,6$ & 3,$865 ; p=0,000$ \\
\hline \multicolumn{4}{|c|}{ Percepção de dificuldades e problemas } \\
\hline \multicolumn{2}{|c|}{ Percepção de muitas dificuldades e problemas } & \multicolumn{2}{|c|}{ Sem percepção de dificuldades e problemas } \\
\hline Ansiedade & $7,4 \pm 3,8$ & $9,1 \pm 4,6$ & N.S. \\
\hline Depressão & $3,3 \pm 2,9$ & $4,8 \pm 3,9$ & N.S. \\
\hline Qvp & $3,8 \pm 3,6$ & $3,5 \pm 0,7$ & N.S. \\
\hline \multicolumn{4}{|c|}{ Percepção de mudanças } \\
\hline \multicolumn{2}{|c|}{ Maior percepção de mudanças } & \multicolumn{2}{|c|}{ Menor percepção de mudanças } \\
\hline Ansiedade & $8,5 \pm 4,4$ & $5,5 \pm 4,8$ & 2,$572 ; p=0,01$ \\
\hline Depressão & $4,1 \pm 3,8$ & $3,0 \pm 3,8$ & N.S. \\
\hline Qvp & $3,7 \pm 0,7$ & $3,8 \pm 0,6$ & N.S. \\
\hline \multicolumn{4}{|c|}{ Informação } \\
\hline \multicolumn{2}{|c|}{ Maior informação } & \multicolumn{2}{|c|}{ Menor informação } \\
\hline Ansiedade & $7,0 \pm 4,7$ & $7,7 \pm 3,7$ & N.S. \\
\hline Depressão & $3,6 \pm 3,6$ & $3,9 \pm 4,3$ & N.S. \\
\hline Qvp & $3,7 \pm 0,6$ & $3,7 \pm 0,9$ & N.S. \\
\hline
\end{tabular}

Qvp - Qualidade de Vida percebida

N.S. - Não significativo 
ção voluntária do tratamento, que resulta em piora do estado de saúde (ora como conseqüência da resistência do vírus ao medicamento ingerido em doses insuficientes ou descontroladas, ora por interrupção do tratamento), as quais, por sua vez redundam em mais percepções negativas.

Por outro lado, consistente com estudos anteriores (Leserman \& cols., 1999; Martínez-Donate \& cols., 2000; Remor \& cols., 2001; Remor, 2002), observou-se que a disponibilidade de apoio social é um fator atenuador do impacto negativo da soropositividade na vida das pessoas afetadas, sendo este um recurso pessoal externo que pode facilitar a adaptação ao processo da doença. A ampliação da rede de apoio e o treinamento de melhores habilidades de comunicação podem ser estratégias úteis para melhorar o apoio social das pessoas com um déficit neste recurso.

À guisa de conclusão, poderíamos dizer que uma dicotomia nas percepções dos pacientes parece evidente. Por um lado estão os pacientes que se adaptaram bem ao tratamento e puderam beneficiar-se destes, e por outro aqueles que não obtiveram os resultados esperados, experimentando incerteza, desesperança e sofrimento, com insatisfação na qualidade de suas vidas.

O estudo dos tratamentos farmacológicos da infecção pelo HIV indica que aproximadamente $50 \%$ dos pacientes não respondem da forma esperada aos tratamentos HAART, e estas porcentagens podem aumentar com o tempo (Deeks, Loftus, Cohen, Chin \&Grant, 1997). Nesse sentido, os conhecimentos da progressão do HIV e seus tratamentos evoluíram muito na curta história da epidemia da Aids, e continuam em constante evolução. Conseqüentemente, as necessidades psicológicas das pessoas soropositivas também vêm mudando. Seguir identificando os temas que afetam a saúde mental dessas pessoas pode contribuir para o desenvolvimento de novas intervenções psicológicas mais adaptadas à realidade da Aids e às necessidades daqueles que vivem com o HIV.

\section{Referências}

Broadhead, W.E., Gehlbach, S.H., Degruy, F.V. \& Kaplan, B.H. (1988). The Duke-UNC functional social support questionnaire: measurement of social support in family medicine patients. Medical Care, 26, 709-723.

Catalan, J., Meadows, J. \& Douzenis, A. (2000). The changing pattern of mental health problems in HIV infection: the vew from London, UK. AIDS Care, 12 (3), 333-341.

Deeks, S.G., Loftus, R., Cohen, P., Chin, S. \& Grant, R. (1997). Incidence and predictors of virological failure of indinavir and/ or ritonavir in an urban health clinic [Resumo LB-02]. Em Program and Abstracts of the 37th Interscience Conference on Antimicrobial Agents and Chemotherapy (p. 286). Toronto, Ontario.

Ferrando, S. (1998). Behavioral Research on AIDS - Protease inhibitors and the new millenium: Comment on Kelly, Otto-Salaj, Sikkema, Pinkerton and Bloom. Health Psychology, 17 (4), 307309.

Furtado, MR., Callaway, DS. \& Phair, JP. (1999). Persistence of HIV-1 transcription in peripheral-blood mononuclear cells in patients receiving potent antiretroviral theraphy. New England Journal of Medicine, 340, 1614-1622.

Guardiola, J.M. \& Soriano, V. (2000). Tratamiento de la infección por VIH-SIDA. Fármacos e combinaciones. Barcelona: Publicaciones Permanyer. $3^{\mathrm{a}}$ ed.

Kelly, J.A., Otto-Salaj, L.L., Sikkema, K.J., Pinkerton, S.D. \& Bloom, F.R. (1998). Implications of HIV treatment advances for behavioral research on AIDS: Protease inhibitors and new challenges in HIV secondary prevention. Health Psychology, 17 (4): 310-319.

Leserman, J., Jackson, E., Petitto, J.M., Golden, R.N., Silva, S.G., Perkins, D.O., Cai, J., Folds, J.D. \& Evans, D.L. (1999). Progression to AIDS: The effects of stress, depressive symptoms, and social support. Psychosomatic Medicine, 61, 397-406.

Martínez-Donate, A., Ortiz, F., Remor, E., Arranz, P., Coca, C., Jiménez, V. \& Hernández-Navarro, F. (2000). Apoyo social y progresión del VIH. Trabalho apresentado no XXX Congress of the European Association for Behavior and Cognitive Therapies, Granada, Espanha.

Nájera, R. (1997). Resistencias a los antirretrovirales de uso clínico. En: J.M. González-Lahoz (org.). Terapia antirretroviral (pp. 48-66). Madrid: SEISIDA.

Ortiz, F., Martinez-Donate, A., Remor, E., Arranz, P., Bayés, R. \& Hernández-Navarro, F. (2000). Estudio comparativo de la adhesión terapeutica en pacientes VIH+ de larga evolución con y sin coagulopatias congénitas. Psiquis, 21 (5), 241-250.

Remor, E.A. (2000). Infección por VIH y SIDA: Características psicológicas y adhesión al tratamiento. Tese de Doutorado, Universidad Autónoma de Madrid, Madrid.

Remor, E. (2002). Apoyo social y calidad de vida en la infección por el VIH. Atención Primaria, 30: 143-148.

Remor, E., Moreno, C., Arranz, P., Ulla, S., Carrobles, J.A., Ramos, J.L., Coca, C., López-Parra, D., Jiménez-Yuste, V. \& Hernández-Navarro, F. (2001). Emociones negativas en la convivencia con el VIH: Estudio comparativo entre pacientes con coagulopatías congénitas y sin coagulopatías congénitas. Trabalho apresentado na XLIII Reunião Nacional da AEHH e XVII Congresso Nacional da SETH, La Coruña, Espanha.

Siegel, K., Schrimshaw, E.W. \& Dean, L. (1999). Symptom interpretation and medication adherence among late middle-age and older HIV-infected adults. Journal of Health Psychology, 4 (2), 247-257.

Ulla, S. \& Remor, E.A. (2002). Psiconeuroimunología e Infecção por HIV: Realidade ou Ficção? Psicología: Reflexão e Crítica, 15 (1), 113-119.

Vallely, P.J., Mani, G.S., Stoddart, R.W., Cleator G.M. \& Lucas, G.S. (1996). Analysis of an HIV-infected cohort followed for as long as 15 years after seroconversion. Journal of Adquired Immune Deficiency Symdrom and Human Retrovirology, 12, 394-399.

Zigmond, A.S. \& Snaith, R.P. (1983). The hospital anxiety and depression scale. Acta Psychiatrica Scandinavica, 67, 361370. 